\title{
Generation of Continuous Variable Einstein-Podolsky-Rosen Entanglement via the Kerr Nonlinearity in an Optical Fiber
}

\author{
Ch. Silberhorn, ${ }^{1}$ P. K. Lam, ${ }^{1,2}$ O. Weiß, ${ }^{1}$ F. König, ${ }^{1}$ N. Korolkova, ${ }^{1}$ and G. Leuchs ${ }^{1}$ \\ ${ }^{1}$ Zentrum für Moderne Optik, Universität Erlangen-Nürnberg, Staudtstraße 7/B2, D-91058 Erlangen, Germany \\ ${ }^{2}$ Department of Physics, Faculty of Science, The Australian National University, ACT 0200, Canberra, Australia
}

(Received 3 August 2000)

\begin{abstract}
We report on the generation of a continuous variable Einstein-Podolsky-Rosen (EPR) entanglement using an optical fiber interferometer. The Kerr nonlinearity in the fiber is exploited for the generation of two independent squeezed beams. These interfere at a beam splitter and EPR entanglement is obtained between the output beams. The correlation of the amplitude (phase) quadratures is measured to be $4.0 \pm 0.2(4.0 \pm 0.4) \mathrm{dB}$ below the quantum noise limit. The sum criterion for these squeezing variances $0.80 \pm 0.03<2$ verifies the nonseparability of the state. The product of the inferred uncertainties for one beam $(0.64 \pm 0.08)$ is well below the EPR limit of unity.
\end{abstract}

DOI: $10.1103 /$ PhysRevLett.86.4267

Since the original proposal of a Gedanken experiment intending to show the incompleteness of quantum mechanics in 1935 [1], a number of schemes for generating the Einstein-Podolsky-Rosen (EPR) entanglement have been realized. The schemes range from the production of gamma-ray pairs from positron-electron annihilations [2], to proton pairs [3], to pairs of low-energy photons from atomic radiative cascade [4], and more recently to schemes involving optical parametric processes [5]. Most of these initial experiments utilized the entanglement as originally intended: to test the validity of quantum mechanics via either the violation of Bell inequality [4] or the demonstration of the EPR paradox [6]. Following the proposals of a myriad of quantum information schemes in recent years where entanglement is regarded as a basic requisite, the subject matter has experienced a resurgence of interest. The purposes of entanglement generation are now shifting to that of quantum information applications. Among these applications are the realization of quantum teleportation, the implementation of dense coding, quantum cryptography, and other quantum communication schemes [7]. In view of these changing needs, it is desirable to explore simpler and more reliable alternatives for the generation of EPR entanglement.

In this Letter, we report on what is to our knowledge the first generation of EPR entanglement of photons that does not rely on any pair production process, such as those in the above-mentioned examples. Instead, the $\operatorname{Kerr}\left(\chi^{(3)}\right)$ nonlinearity of an optical fiber is utilized to produce two amplitude squeezed beams, with the nonlinear interaction of each beam uncoupled to the other. To create the EPR entanglement, no additional nonlinear interaction is required. Instead, the amplitude squeezed beams are made to interfere at a 50/50 beam splitter [8]. In this vein sum squeezing is obtained for the amplitude quadratures and difference squeezing for the phase quadratures. The signs of these correlations are interchanged compared to those achieved in other systems. This fact may be of importance in applications involving the optomechanical coupling of
PACS numbers: 42.50.Dv, 03.65.Ta, 03.67.-a, 42.65.Tg

radiation pressure [9]. Apart from the simplicity of our scheme, it also has the potential advantage of being integrable into existing fiber-optics communication networks.

Our experimental setup is shown in Fig. 1. A passively mode-locked $\mathrm{Cr}^{4+}$ :YAG (yttrium-aluminum-garnet) laser is used to produce optical pulses at a center wavelength of $1505 \mathrm{~nm}$ with a repetition frequency of $163 \mathrm{MHz}$. The maximum average power of the laser is around $95 \mathrm{~mW}$ and the pulses have a bandwidth limited hyperbolicsecant shape with a FWHM of 130 fs. These pulses are injected into an asymmetric fiber Sagnac interferometer. The Sagnac loop consists of an $8 \mathrm{~m}$ long polarization maintaining fiber (FS-PM-7811 from 3M) and a beam splitter. The fiber has a birefringence, characterized by a beat length of $1.95 \mathrm{~mm}$ for $1505 \mathrm{~nm}$ light, that supports the $s$ - and $p$-polarization states with negligible cross talk. The beam splitter of the interferometer has $91 \%(90 \%)$ reflectivity and $9 \%(10 \%)$ transmittivity for the $s(p)$-polarization

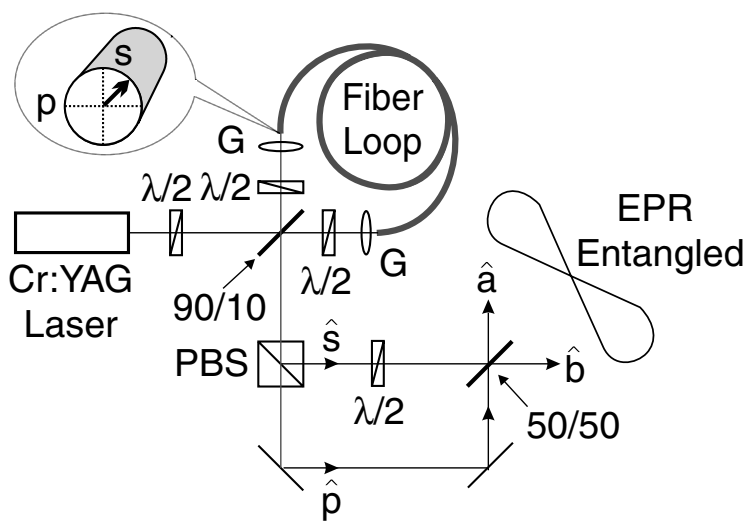

FIG. 1. Schematic of the experimental setup. $\lambda / 2$ : half-wave plate, G: gradient index lens, PBS: polarizing beam splitter, 50/50: beam splitter with 50\% reflectivity; and 90/10: beam splitter with $90 \%$ reflectivity. $\hat{s}$ and $\hat{p}$ are the two squeezed beams from the respective polarization states. $\hat{a}$ and $\hat{b}$ are the EPR entangled output beams. Inset shows the polarization direction of the input beam to the fiber. 
states, respectively. This provides a strong and a weak counterpropagating pulse within the Sagnac loop, one pair for each polarization.

Because of the Kerr nonlinearity of the optical fiber, the strong pulses acquire an intensity dependent phase shift during propagation. This mechanism squeezes the circular shaped phase space uncertainty into an ellipse [10]. However, the squeezed quadrature of the ellipse is not aligned with the amplitude quadrature and amplitude squeezing is not readily observed. In the asymmetric Sagnac interferometric arrangement, the weak and the strong counterpropagating pulses acquire different nonlinear phase shifts. When the pulses interfere at the beam splitter, this relative phase shift realigns the axes of the ellipse. For certain input energies, this realignment yields direct detectable amplitude squeezing [11].

In contrast to the setup reported by Schmitt et al. [11], however, we utilized the polarization maintaining characteristics of the fiber to simultaneously sustain two orthogonally polarized modes in the Sagnac loop by choosing an input polarization direction at around $45^{\circ}$ relative to the fiber axes. Thus, two independently amplitude squeezed beams, labeled as $\hat{s}$ and $\hat{p}$ in Fig. 1, are produced.

Owing to the birefringence of the optical fiber, the $\hat{s}$ - and $\hat{p}$-polarization modes of the same energy in general do not experience the same effective Kerr nonlinearity. This is because both the propagation time and the mode confinement for each polarization are different. Furthermore, the reflectivity of the Sagnac mirror is slightly different for the two polarizations. As a result, simultaneous squeezing of both modes is not always guaranteed. This imbalance can be compensated by careful adjustment of the energy splitting ratio between the two polarizations.

The photodetectors used are a pair of balanced windowless InGaAs detectors from Epitaxx (ETX-500). The detection efficiencies are measured to be around $(92 \pm 5) \%$ and the detection frequency is at $10 \mathrm{MHz}$ with a resolution bandwidth of $300 \mathrm{kHz}$. Our detectors have their $3 \mathrm{~dB}$ roll-off frequencies at around $20 \mathrm{MHz}$ and a more than $40 \mathrm{~dB}$ attenuation at the repetition frequency and the harmonics of the mode-locked $\mathrm{Cr}^{4+}$ :YAG laser. In order to obtain an EPR entanglement, the polarization of the $\hat{s}$ beam and its path length are adjusted to interfere with the $\hat{p}$ beam at a $50 / 50$ beam splitter.

For two squeezed sources with identical squeezed quadrature and optical power, EPR entanglement is maximized when the interference phase is such that the two output beams have equal optical power [12]. We can model this condition by assuming that both reflected beams have $90^{\circ}$ phase shifts relative to their transmitted beams. In order to evaluate the noise variance expressions of the output beams, we express the annihilation operator of a field as $\hat{A}(t)=\alpha+\delta \hat{A}(t)$, with $\alpha$ being the classical steady state value, and $\delta \hat{A}(t)$ the zero-mean operator which contains all of the classical and quantum fluctuations. We denote the amplitude and phase quadratures with $\hat{X}^{+}(t)=\hat{A}^{\dagger}(t)+$ $\hat{A}(t)$ and $\hat{X}^{-}(t)=i\left[\hat{A}^{\dagger}(t)-\hat{A}(t)\right]$, respectively. Finally,

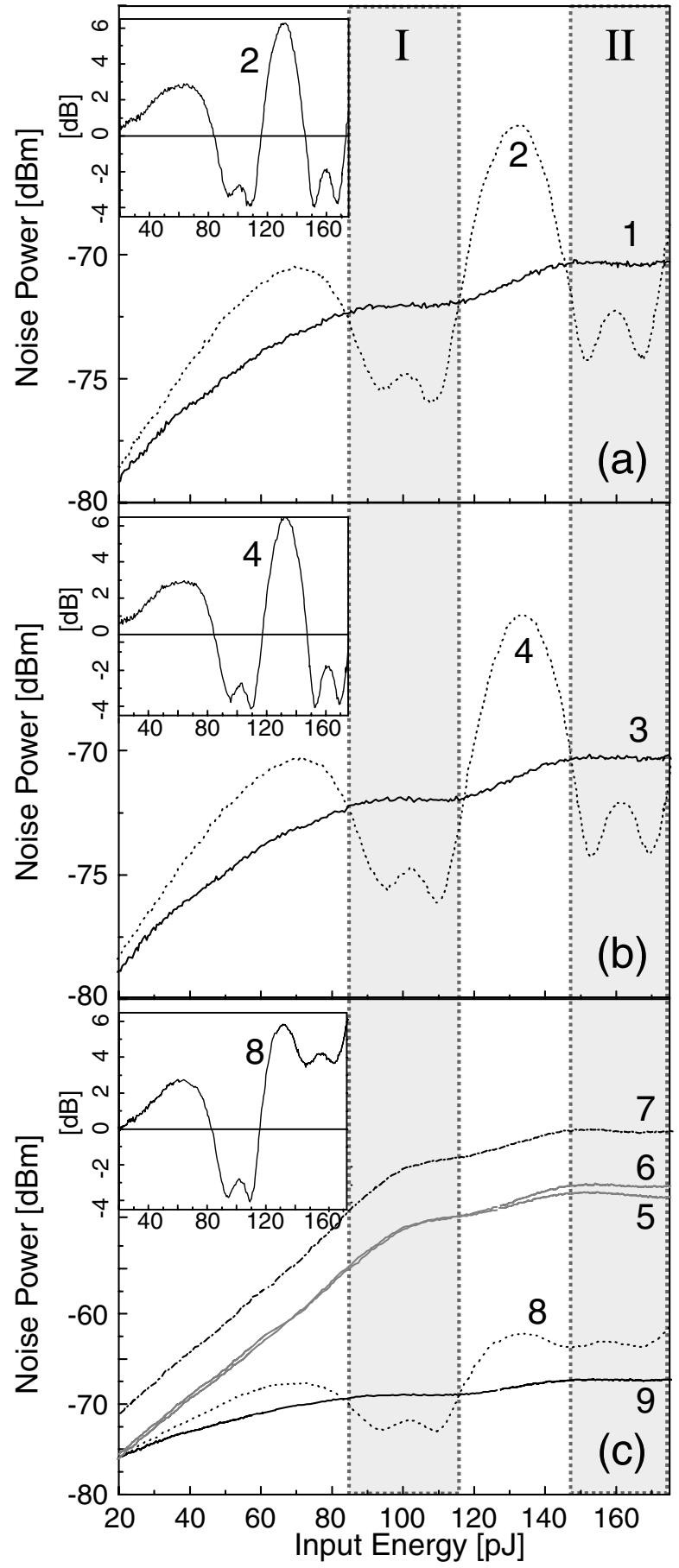

FIG. 2. Experimental results. (a),(b) Squeezing as a function of input pulse energy for the $s(p)$-polarized beams, respectively. (c) Noise measurements when both squeezed beams are made to interfere at the $50 / 50$ beam splitter. Insets show the normalized variances of the respective traces. Region I gives the energy range with EPR entangled outputs; in region II both input beams appear squeezed, but no quantum correlations are measured at the outputs (see text).

via a Fourier transform, the spectral variances $V\left(\hat{X}_{A}^{ \pm}\right)=$ $V\left(\hat{X}_{A}^{ \pm}\right)(\omega)=\left\langle\left|\delta \hat{A}(\omega) \pm \delta \hat{A}^{\dagger}(\omega)\right|^{2}\right\rangle$ are calculated by considering only the first order contribution of the fluctuation terms. The noise variances of the output beams $\hat{a}$ 
and $\hat{b}$ are equal to

$$
V\left(\hat{X}_{a, b}^{+}\right)=\frac{1}{4}\left[V\left(\hat{X}_{s}^{+}\right)+V\left(\hat{X}_{s}^{-}\right)+V\left(\hat{X}_{p}^{+}\right)+V\left(\hat{X}_{p}^{-}\right)\right],
$$

and the normalized sum and difference photocurrents for both amplitude and phase quadratures are then given by

$$
\begin{aligned}
& V_{\mathrm{sum}}^{ \pm}=\frac{V\left(\hat{X}_{a}^{ \pm}+\hat{X}_{b}^{ \pm}\right)}{V\left(\hat{X}_{a, \mathrm{SN}}^{ \pm}+\hat{X}_{b, \mathrm{SN}}^{ \pm}\right)}=\frac{1}{2}\left[V\left(\hat{X}_{s}^{ \pm}\right)+V\left(\hat{X}_{p}^{ \pm}\right)\right], \\
& V_{\mathrm{diff}}^{ \pm}=\frac{V\left(\hat{X}_{a}^{ \pm}-\hat{X}_{b}^{ \pm}\right)}{V\left(\hat{X}_{a, \mathrm{SN}}^{ \pm}-\hat{X}_{b, \mathrm{SN}}^{ \pm}\right)}=\frac{1}{2}\left[V\left(\hat{X}_{s}^{\mp}\right)+V\left(\hat{X}_{p}^{\mp}\right)\right],
\end{aligned}
$$

with the field modes denoted by the respective subscripts, and $\mathrm{SN}$ is used to denote the corresponding parameter for a shot noise limited beam. For amplitude squeezed input beams, we have $V\left(\hat{X}_{s, p}^{+}\right)<1<V\left(\hat{X}_{s, p}^{-}\right)$, thus giving sum squeezing for the amplitude quadrature, $V_{\text {sum }}^{+}<1$, and difference squeezing for the phase, $V_{\text {diff }}^{-}<1$. Apart from this, we note that all other variances for the output beams are above the quantum noise limit.

Our initial experimental investigation is done by varying the input pulse energy continuously while measuring the $\hat{s}$ and $\hat{p}$ output beams individually using a balanced detection scheme. The results of these measurements are shown in Figs. 2(a) and 2(b). Traces 1 and 3 are the respective quantum noise limits and traces 2 and 4, the amplitude noise variances. We observed that similar to [11], the amplitude noise power varies as a function of the input pulse energy giving rise to squeezing regions I and II. This is a consequence of the variation in the nonlinear phase shift experienced as a function of pulse energy. The noise power traces have a double-dip structure within a squeezing region due to the nonoptimal reflectivity of the Sagnac beam splitter [11]. We note that the squeezing regions of these two different polarization beams overlap almost completely. The maximum amplitude squeezing obtained in region $\mathrm{I}$ is $4.1 \pm 0.2 \mathrm{~dB}(3.9 \pm 0.2 \mathrm{~dB})$ for the $p(s)$-polarization beam.

In our experiment the beam splitter transforms the two squeezed input beams into quantum entanglement between the output beams. The efficiency of this process relies on high interferometric visibility. For the input energies of region I a visibility of $96 \% \pm 1 \%$ was measured from a beam splitter with $51.5 \% \pm 1.0 \%$ reflectivity. After the interference the outputs are measured individually. In Fig. 2(c), traces 5 and 6 show the amplitude noise variances of the $\hat{a}$ and $\hat{b}$ EPR beams, respectively. As
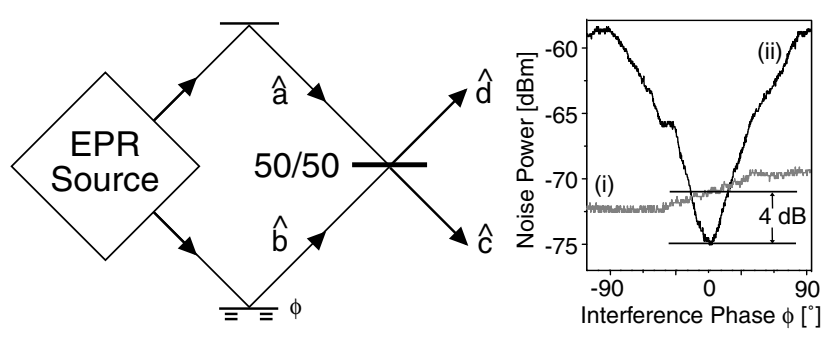

FIG. 3. Left: Schematic for the indirect interrogation of the phase quadrature correlation. Right: Noise variance of the output beam $\hat{c}$ when the interferometer phase is scanned. (i) Quantum noise limit, (ii) noise variance of $\hat{c}$.

predicted by Eq. (1), the noise variances of the individual beams are far above the quantum noise limit since each of them contains the squeezed and the antisqueezed noise variances of both the $\hat{s}$ and $\hat{p}$ beams. Trace 7 shows that the noise power obtained when both beams are detected and subtracted is $6 \mathrm{~dB}$ above that of the individual EPR beams (traces 5 and 6). This is because it is measured at twice the optical power of an individual EPR beam $(3 \mathrm{~dB})$ and $V_{\text {diff }}^{+} \approx 2 V\left(X_{a, b}^{+}\right)$(a further $3 \mathrm{~dB}$ ).

The excess noise is present in the variances of each individual beam (traces 5 and 6 ), but the variance of the sum photocurrents (trace 8) is observed to be below the quantum noise limit (trace 9). The best result obtained in our setup is found at the input energy near $110 \mathrm{pJ}$ with $4.0 \pm 0.2 \mathrm{~dB}$ of quantum correlation. Although there is squeezing of both input beams in region II, the sum variance of the amplitudes is above the quantum noise limit. This is attributed to the following reasons. First there is a lack of cancellation from the balanced detector pair due to nonlinear responses at input energy beyond $130 \mathrm{pJ}$. Moreover, the interferometric visibility is optimized only for region I at the input energy of around $110 \mathrm{pJ}$. At higher input energies, stimulated Raman scattering begins to dominate, which leads to a decrease in the spectral overlap between the beams. Hence, EPR entanglement is present only in region I (see Fig. 2).

In order to directly measure the phase quadrature correlation of the output beams, optical local oscillators are required for homodyne measurements. In our experiment, this is not possible due to pump power limitation and detector saturation. Instead, an indirect interrogation of the phase quadrature correlation as shown in Fig. 3 is devised. Let us assume that the experiment described thus far is a black box with two output beams of which the amplitude correlation has been established. We now let these beams interfere at yet another 50/50 beam splitter. The final output beams are now denoted by $\hat{c}$ and $\hat{d}$ and their variances are given by

$$
\begin{aligned}
V\left(\hat{X}_{c, d}^{+}\right)= & \frac{1}{4}\left\langle\left(\delta \hat{X}_{a}^{+}+\delta \hat{X}_{b}^{+}+\delta \hat{X}_{a}^{-}-\delta \hat{X}_{b}^{-}\right)^{2}\right\rangle \\
= & \frac{1}{4}\left[V\left(\hat{X}_{a}^{+}+\hat{X}_{b}^{+}\right)+V\left(\hat{X}_{a}^{-}-\hat{X}_{b}^{-}\right)\right] \\
& \pm\left(\left\langle\delta \hat{X}_{a}^{+} \delta \hat{X}_{a}^{-}\right\rangle-\left\langle\delta \hat{X}_{b}^{+} \delta \hat{X}_{b}^{-}\right\rangle+\left\langle\delta \hat{X}_{b}^{+} \delta \hat{X}_{a}^{-}\right\rangle-\left\langle\delta \hat{X}_{a}^{+} \delta \hat{X}_{b}^{-}\right\rangle\right)
\end{aligned}
$$


By argument of symmetry, we note that $\hat{a}$ and $\hat{b}$ are produced similarly and interchanging the indices should not give a different result. The terms within the bracket in the third line of Eq. (4) therefore should cancel each other. Hence measurements of the amplitude variances of $\hat{c}$ and $\hat{d}$ are equivalent to combined measurements of the amplitude sum variance, $V\left(\hat{X}_{a}^{+}+\hat{X}_{b}^{+}\right)$, and the phase difference variance, $V\left(\hat{X}_{a}^{-}-\hat{X}_{b}^{-}\right)$. Since $V\left(\hat{X}_{a}^{+}+\hat{X}_{b}^{+}\right)$is known from earlier measurements, we can therefore deduce $V\left(\hat{X}_{a}^{-}-\hat{X}_{b}^{-}\right)$without using a separate local oscillator. With this method we measured a best difference squeezing of $V_{\text {diff }}^{-}=4.0 \pm 0.4 \mathrm{~dB}$ for the phase quadratures at the input pulse energy of $110 \mathrm{pJ}$, in excellent agreement with the prediction of Eq. (3). The result of a typical scan of this second interferometer phase is shown in Fig. 3. The noise suppression predicted by Eq. (4) is observed only at the interferometer phase of $\phi=0$, corresponding to $\hat{c}$ and $\hat{d}$ having equal power.

In the experiment the quantum correlations in the two noncommuting observables, amplitude and phase are characterized by the sum $V_{\text {sum }}^{+}=0.40 \pm 0.02$ and difference $V_{\text {diff }}^{-}=0.40 \pm 0.04$ squeezing of the entangled beams where the corresponding variances of the sum and difference photocurrents are normalized to the shot noise level of both beams [Eqs. (2) and (3)]. With this normalization the Peres-Horodecki separability criterion for continuous variables [13] can be expressed as $V_{\text {sum }}^{+}+V_{\text {diff }}^{-}<2$. Thus the value of $V_{\text {sum }}^{+}+V_{\text {diff }}^{-}=0.80 \pm 0.03<2$ shows the high nonseparability of the generated state. For many quantum information applications, this criterion also defines the boundary between the classical and the quantum regime. For example, the limit of the Peres-Horodecki criterion corresponds to a teleportation fidelity of $F=$ $\left\langle\psi_{\text {in }}\left|\rho_{\text {out }}\right| \psi_{\text {in }}\right\rangle=0.5$, which separates classical and quantum teleportation. Assuming our realistic EPR source and ideal conditions for the teleportation, this would yield a fidelity of $F=0.71 \pm 0.02>\frac{2}{3}$ (for criteria of quantum teleportation see [14-16]).

In terms of the EPR Gedanken experiment, one has to apply another criterion. The demonstration of the EPR paradox requires the ability to infer "at a distance" both noncommuting observables with a precision below the quantum noise limit of one single beam [17]. The symmetry between the beams in optical powers and quantum uncertainties allows us to renormalize to obtain the appropriate inferred variances for the amplitude and phase quadrature. These inferred variances for an optimum gain of unity are the conditional variances. Their product $V\left(\hat{X}_{a, \text { cond }}^{+}\right) V\left(\hat{X}_{a, \text { cond }}^{-}\right)=V\left(\hat{X}_{a}^{+}+\hat{X}_{b}^{+}\right) V\left(\hat{X}_{a}^{-}-\hat{X}_{b}^{-}\right)=$ $0.64 \pm 0.08$ is less than unity satisfying the condition for the demonstration of the EPR paradox [6,17].

In summary, we have presented a scheme for the generation of EPR entangled beams using the Kerr nonlinearity of an optical fiber. All results presented are observed quantities not corrected for linear losses, apart from subtracting the electronic noise of the detectors. Moreover, the stability of the EPR entanglement is limited only by the stabil- ity of the pump laser. Since the fiber is the only nonlinear medium used, it has advantages over the existing methods of EPR entanglement generation in terms of its simplicity, cost, and integrability into existing communication networks. There are two possible outlooks for this basic building block for quantum communication. First, there is some prospect to further improve the quality of the entanglement, since more than $11 \mathrm{~dB}$ of amplitude squeezing were predicted with a Sagnac loop even when realistic experimental parameters were considered [11]. Second, the lack of the phase measurement can be overcome by implementing an arm length unbalanced interferometer, which under appropriate conditions converts the phase quadrature variances into directly detectable amplitude variances without introducing additional noise [18]. Then, due to its simplicity, multiple EPR entanglement and other more sophisticated quantum communication schemes can be demonstrated in the future.

We gratefully acknowledge financial support of the Deutsche Forschungsgemeinschaft, of the EU grant under QIPC, (QUICOV), and of the Alexander von Humboldt Foundation (P. K. L.).

[1] A. Einstein, B. Podolsky, and N. Rosen, Phys. Rev. 47, 777 (1935).

[2] C. S. Wu and I. Shaknov, Phys. Rev. 77, 136 (1950).

[3] M. Lamehi-Rachti and W. Mittig, Phys. Rev. D 14, 2543 (1976).

[4] S. J. Freedman and J. F. Clauser, Phys. Rev. Lett. 28, 938 (1972); A. Aspect, J. Dalibard, and G. Roger, Phys. Rev. Lett. 49, 1804 (1982).

[5] See, for example, Y.H. Shih and C. O. Alley, Phys. Rev. Lett. 61, 2921 (1988); P. G. Kwiat, K. Mattle, H. Weinfurter, and A. Zeilinger, Phys. Rev. Lett. 75, 4337 (1995).

[6] Z. Y. Ou, S. F. Pereira, H. J. Kimble, and K. C. Peng, Phys. Rev. Lett. 68, 3663 (1992).

[7] Special issue of Physics World, March (1998).

[8] A. Furusawa, J. L. Sørensen, S. L. Braunstein, C. A. Fuchs, H. J. Kimble, and E. S. Polzik, Science 282, 706-709 (1998).

[9] P. F. Cohadon, A. Heidmann, and M. Pinard, Phys. Rev. Lett. 83, 3174 (1999).

[10] P.D. Drummond, R. M. Shelby, S. R. Friberg, and Y. Yamamoto, Nature (London) 365, 307 (1993).

[11] S. Schmitt, J. Ficker, M. Wolff, F. König, A. Sizmann, and G. Leuchs, Phys. Rev. Lett. 81, 2446 (1998).

[12] G. Leuchs et al., J. Mod. Opt. 46, 1927-1939 (1999).

[13] L.-M. Duan, G. Giedke, J.I. Cirac, and P. Zoller, Phys. Rev. Lett. 84, 2722 (2000); R. Simon, Phys. Rev. Lett. 84, 2726 (2000).

[14] S. L. Braunstein, C. A. Fuchs, and H. J. Kimble, J. Mod. Opt. 47, 267 (2000); S. L. Braunstein, C. A. Fuchs, H. J. Kimble, and P. van Loock, quant-ph/0012001.

[15] T. C. Ralph and P. K. Lam, Phys. Rev. Lett. 81, 5668 (1998).

[16] F. Grosshans and P. Grangier, quant-ph/0012121.

[17] M. D. Reid and P. D. Drummond, Phys. Rev. Lett. 60, 2731 (1988); M. D. Reid, Phys. Rev. A 40, 913 (1989).

[18] P. Grangier and J. Trautner (private communication). 